\title{
Statistical analysis of emission, interaction and annihilation of phonons by kink motion in ferroelastic materials
}

Cite as: Appl. Phys. Lett. 116, 102902 (2020); https://doi.org/10.1063/1.5143977

Submitted: 28 December 2019 . Accepted: 23 January 2020 . Published Online: 13 March 2020

Libo Zhang, Suzhi Li, Xiangdong Ding (D), Jun Sun, and Ekhard K. H. Salje (iD)

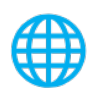

\section{ARTICLES YOU MAY BE INTERESTED IN}

Development of microLED

Applied Physics Letters 116, 100502 (2020); https://doi.org/10.1063/1.5145201

Observing relaxation in device quality InGaN templates by TEM techniques

Applied Physics Letters 116, 102104 (2020); https://doi.org/10.1063/1.5139269

Pitfalls and prospects of optical spectroscopy to characterize perovskite-transport layer interfaces

Applied Physics Letters 116, 100501 (2020); https://doi.org/10.1063/1.5143121

Lock-in Amplifiers Find out more today

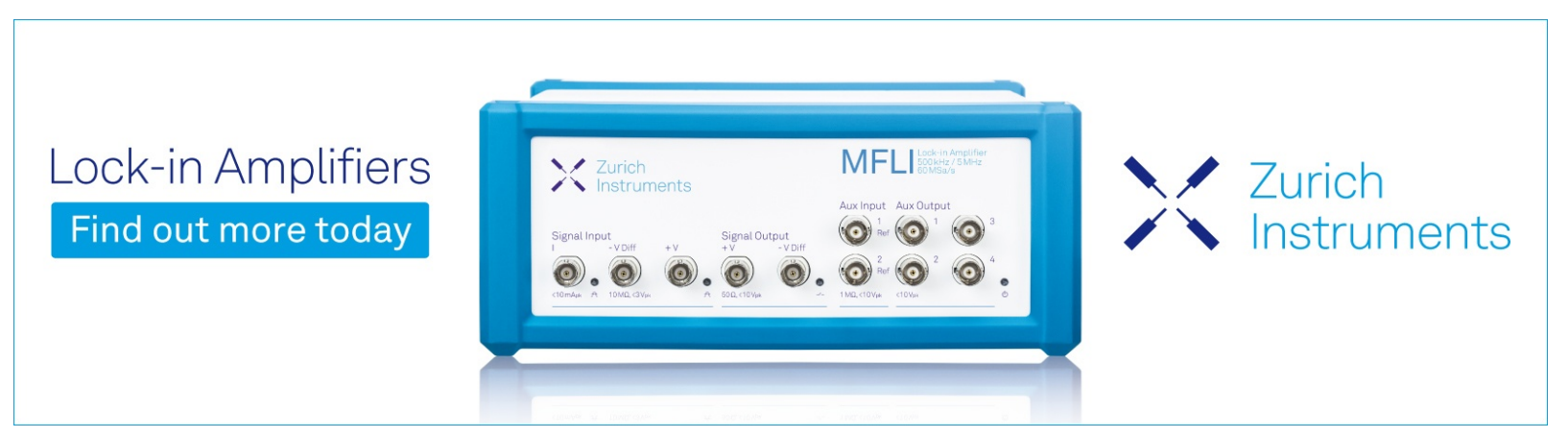

Zurich Instruments 


\title{
Statistical analysis of emission, interaction and annihilation of phonons by kink motion in ferroelastic materials
}

Cite as: Appl. Phys. Lett. 116, 102902 (2020); doi: 10.1063/1.5143977

Submitted: 28 December 2019 - Accepted: 23 January 2020 •

Published Online: 13 March 2020

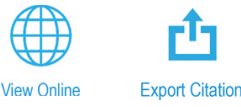

Libo Zhang, ${ }^{\top}$ Suzhi Li, ${ }^{1, a)}$ Xiangdong Ding, ${ }^{1, a)}$ (D) Jun Sun, and Ekhard K. H. Salje ${ }^{1,2, a)}$ (iD

\begin{abstract}
AFFILIATIONS
${ }^{7}$ State Key Laboratory for Mechanical Behavior of Materials, Xi'an Jiaotong University, Xi'an 710049, China

${ }^{2}$ Department of Earth Sciences, University of Cambridge, Cambridge CB2 3EQ, United Kingdom
\end{abstract}

a) Authors to whom correspondence should be addressed: lisuzhi@xjtu.edu.cn; dingxd@mail.xjtu.edu.cn; and ekhard@esc.cam.ac.uk

\begin{abstract}
Our early work showed that the evolution of the twin boundary pattern exhibits an avalanche behavior upon external loading of ferroelastic materials [Salje et al., Phys. Rev. B 83, 104109 (2011)]. The distribution of "jerks" (singularities of potential energy change) was found to follow a power law distribution below a Vogel-Fulcher temperature, mainly related to the movement of kinks in domain boundaries. We use molecular dynamics simulations to study the nucleation, scattering, and annihilation of phonons that are generated by the nucleation and propagation of such kinks. The interaction and scattering of phonons are correlated over a short time period and gradually become uncorrelated before annihilation at large temperature intervals. The movement and interaction of phonons show avalanche behavior. The probability of finding energy jerks follows a power law with exponents around 2.5-3. The distribution of waiting times between jerks also follows a power law. At temperatures above the Vogel-Fulcher temperature, scattering with thermal phonons becomes predominant and no phononic avalanches were observed.
\end{abstract}

Published under license by AIP Publishing. https://doi.org/10.1063/1.5143977

Crackling noise ${ }^{1,2}$ corresponds to the intermittent response to a slowly driven external field. The short-time singularities are termed jerks. The generation of multiple jerky events shows a typical avalanche behavior that is observed in a variety of phenomena, such as martensitic transitions in crystals, ${ }^{3}$ plastic deformation of materials, ${ }^{4,5}$ polarization in ferroelectrics, ${ }^{6}$ etc. Similar statistical laws are found in a variety of dynamic processes in analogy to those in earthquakes. ${ }^{1,7}$ For example, it is found that the avalanche sizes and duration follow a power law distribution spanning a broad range, exhibiting a lack of characteristic length and time scales. ${ }^{8}$ The rate of aftershocks after big jerks decays with time, following the Omori law. ${ }^{9}$ The distribution of waiting time is found to follow a universal scaling law. ${ }^{10}$

Another typical example of crackling noises is the domain switching process in ferroelastic materials upon mechanical deformation. ${ }^{11}$ The characteristic noises stem from the jerky motion of domain walls. Experimentally, the acoustic emission (AE) is widely used to detect the avalanche of domain wall motion and domain switching. ${ }^{3,12}$ Energy distributions ${ }^{8}$ follow power laws with exponents ranging from 1.3 to 2.3 . The exponent values depend on many factors, such as the system size, temperature, microstructure, etc. ${ }^{13}$ For example, the compression tests on porous TiNi reveal that the energy exponent is around 2.0 for detwinning and 1.7 for fracture. ${ }^{12}$ Thus, the statistical analysis on the microstructure change in ferroelastic materials is of fundamental importance in the aspect of "domain boundary engineering" and the disentanglement of the various contributions to domain boundary movements.

Early atomistic simulations studied the effect of temperature on the evolution of domain patterns involving the nucleation, propagation and interactions of needle domains. ${ }^{14,15}$ Energy distribution of avalanches follows the power law in a certain temperature range below the Vogel-Fulcher temperature $\left(\mathrm{T}_{\mathrm{VF}}\right)$ in a thin ferroelastic film with an exponent around 2.0, ${ }^{14}$ which is associated with the jamming and subsequent progression process of needle domains. Waiting time distribution also obeys the power law for a modest temperature below $\mathrm{T}_{\mathrm{VF} .}^{2}$

More fundamentally, the kink-pair mechanism is responsible for the propagation of the domain wall. ${ }^{2,11,14,16-18}$ Phonon emission is associated with kink nucleation and propagation. At low temperatures, 
these mechanically generated phonons that interact, scatter, and finally annihilate. However, little has been done to explore these phononic avalanches. In this work, using atomistic simulations, we studied the nucleation, motion, and annihilation of phonons generated by nucleation and propagation of a kink pair at a twin boundary under external loading. The energy jerks follow a power law distribution at $0.1 \mathrm{~K}-5 \mathrm{~K}$ with exponents ranging from 2.5 to 3.0 at temperature below the Vogel-Fulcher point. Event rates decay according to Omori's law, then remain unchanged, and finally drop down quickly. At a higher temperature, the thermal phonon scattering becomes dominant and no phononic avalanches were observed.

We use a two-body potential based on the Landau theory to describe the interactions in atoms. ${ }^{14}$ The potential energy $U(r)$ consists of four interactions, ${ }^{19}$ the first-nearest atomic interactions $\mathrm{U}_{\mathrm{NN}}$ $=20(\mathrm{r}-1)^{2}(0.8 \leq \mathrm{r} \leq 1.2)$, the second-nearest atomic interactions $\mathrm{U}_{\mathrm{NNN}}=-10(\mathrm{r}-\sqrt{2})^{2}+8000(\mathrm{r}-\sqrt{2})^{4}(1.207 \leq \mathrm{r} \leq 1.621)$, the third-nearest atomic interactions $\mathrm{U}_{\mathrm{NNNN}}=8(\mathrm{r}-2)^{4} \quad(1.85 \leq \mathrm{r}$ $\leq 2.15)$, and the fourth-nearest atomic interactions $U_{\mathrm{NNNNN}}=-10(\mathrm{r}$ $-\sqrt{5})^{2}+5100(\mathrm{r}-\sqrt{5})^{4}(2.15<\mathrm{r} \leq 2.32)$, where $\mathrm{r}$ is the atomic distance vector. The shear angle is the order parameter in this model. The equilibrium unit cell of the ferroelastic domain is a parallelogram with a shear angle of $2^{\circ}$. We set the equilibrium lattice constant $a=1$ and atomic mass $\mathrm{M}=10 \mathrm{amu}$. The simulation box size is $40 a \times 42 a$ $(\sim 4 \mathrm{~nm} \times 4.2 \mathrm{~nm})$. The initial sandwich sample contains two preexisting horizontal twin boundaries. We set the ratio of the height of the intermediate layer to the whole sample to be 0.5 . Free boundary conditions are adopted. Initially, the system was relaxed via the conjugate gradient refinement procedure. Then, dynamic relaxation was performed to anneal the sample at the temperatures ranging from $\mathrm{T}=0.1 \mathrm{~K}\left(5 \times 10^{-3} \mathrm{~T}_{\mathrm{VF}}\right)$ to $\mathrm{T}=10 \mathrm{~K}\left(0.5 \mathrm{~T}_{\mathrm{VF}}\right)$, where $\mathrm{T}_{\mathrm{VF}}$ $=20 \mathrm{~K}$ is the Vogel-Fulcher temperature. After relaxation, we apply the external shear strain via top and bottom fixed layers. The strain rate is around $5 \times 10^{8} \mathrm{~s}^{-1}$. A canonical ensemble (NVT) is used and the temperature is kept by a Nosé-Hoover thermostat. ${ }^{20}$ All the MD simulations were performed with the LAMMPS code. ${ }^{21}$

Figure 1(a) shows the variation of the potential energy $(\mathrm{Pe})$ as a function of the shear strain $\left(\mathrm{e}_{\mathrm{s}}\right)$ in a sandwich twin at $1 \mathrm{~K}$. The system first undergoes an elastic deformation with the potential energy increasing quadratically. After yielding, the potential energy curve exhibits a saw-tooth behavior over the whole plastic regime. Figure (b) shows the big energy drop in one periodicity marked in Fig. 1(a). It corresponds to a big energy jerk $\mathrm{E}$, which is characterized as the square of derivative of potential energy with strain $(\mathrm{dPe} / \mathrm{de})^{2}$. After the big jerk, many small jerks are generated, as shown in Fig. 1(c).

To understand the origin of jerks, we checked the evolution of microstructure in one periodicity. Figures $1(\mathrm{~d})-1(\mathrm{k})$ shows the typical atomic images at times marked in Figs. 1(b) and 1(c). Near the peak, a pair of kinks nucleates at the upper twin boundary [Fig. 1(d)], and then propagates along the domain boundary [Fig. 1(e)]. The two kinks finally annihilate at the free surfaces [Fig. 1(f)], leading to the movement of the upper twin boundary by one atomic layer. The big jerk is generated with a magnitude of $\mathrm{E}>10^{11} \mathrm{eV}^{2}$ when the kink nucleation occurs. During the kink movement, we observe that phonons are emitted [Fig. 1(e)]. When the kinks hit the surfaces, additional waves are
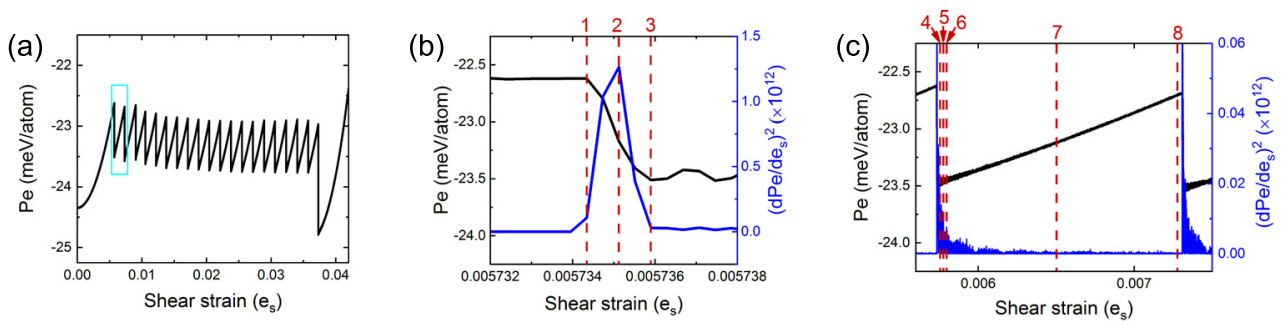

(d)

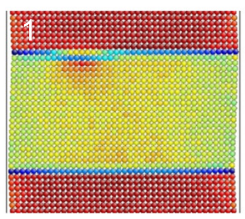

(e)

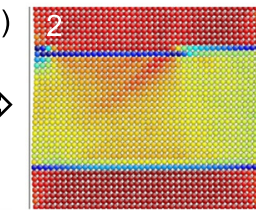

(h)

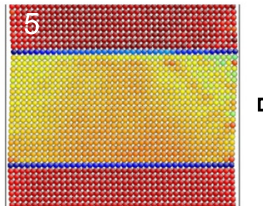

(i)

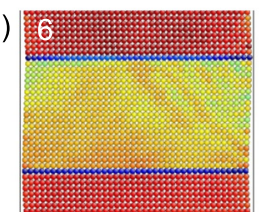

(f)
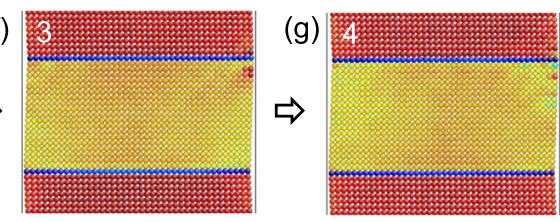

(j)

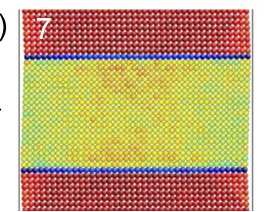

(k)

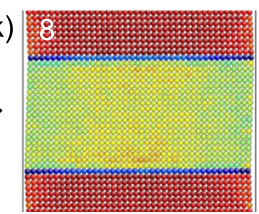

0.33

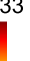

$-2.12$

FIG. 1. Mechanically driven kink motion under shear deformation in a twinned structure at $1 \mathrm{~K}$. (a) Potential energy as a function of external shear strain showing the saw-tooth profiles. (b) Jerk spectrum around the energy peak in one periodicity. (c) Jerk spectrum between two periodicities. (d)-(k) The typical atomic images as marked in (b) and (c). The color is coded by the local shear angle. (d)-(f) The big energy drop in potential energy corresponds to a kink pair nucleation, propagation, and interaction with the surfaces. (e)-(h) Kink motion and kink-surface interactions emit waves associated with the following avalanches. (g)-(i) Propagation and interaction of waves in crystals after the kink annihilation. (j)-(k) The waves diminish after relaxation until another kink is generated when the potential energy approaches the next peak. The color shows the local shear angle $\theta=\left|\theta_{\text {ver }}\right|+\theta_{\text {hor }}-2^{\circ}$, where $\theta_{\text {ver }}$ and $\theta_{\text {hor }}$ donate the local shear angle in the vertical direction and horizontal direction, respectively. 
generated near the surfaces [Figs. 1(g)-1(i)]. These waves propagate, interact, and gradually diminish after relaxation [Fig. 1(j)]. This effect produces several small jerks with a magnitude 2-4 orders lower than the big jerks. Further loading leads to an accumulation of elastic strain energy until it reaches the critical value to generate another pair of kinks [Fig. 1(k)]. This process, i.e., kink nucleation, propagation, and annihilation, repeats itself, making the whole potential energy vary periodically until the detwinning process is completed. We estimated the critical local shear strain $\left(\varepsilon_{\mathrm{xy}}{ }^{\text {critical }}\right)$ for generating kinks. The $\varepsilon_{\mathrm{xy}}{ }^{\text {critical }}$ is around 0.0125 at $\mathrm{T}=0.1 \mathrm{~K}$ and decreases with the increase in temperature (see the supplementary material, Fig. S1). We also calculated the activation barrier for kink nucleation (see the supplementary material, Fig. S2).

We carry out statistical analysis on the jerk spectrum. We filter out the big jerks generated by kink nucleation [Fig. 1(b)] and only consider the jerks signals produced by the propagation and interaction of phonons emitted by the kink motion [Fig. 1(c)]. As observed in the potential energy-strain curves and the corresponding energy spectrum at different temperatures (see the supplementary material, Fig. S3), the jerk size and magnitude of $\mathrm{E}$ become smaller as the temperature decreases. Figure 2(a) shows the probability distribution function (PDF) of energy jerks after the main peak. The PDF of energy is power law distributed in an interval between $\mathrm{T}=0.1 \mathrm{~K}$ and $5 \mathrm{~K}$. The power law exponent is near 2.5-3. The exponential cut-off becomes dominant at higher temperature $(10 \mathrm{~K})$. The data points with high jerk energies ( $\log \mathrm{E} \sim 12)$ correspond to jerky signals for kink nucleation and not phonon motions. Figure 2(b) shows the maximum likelihood
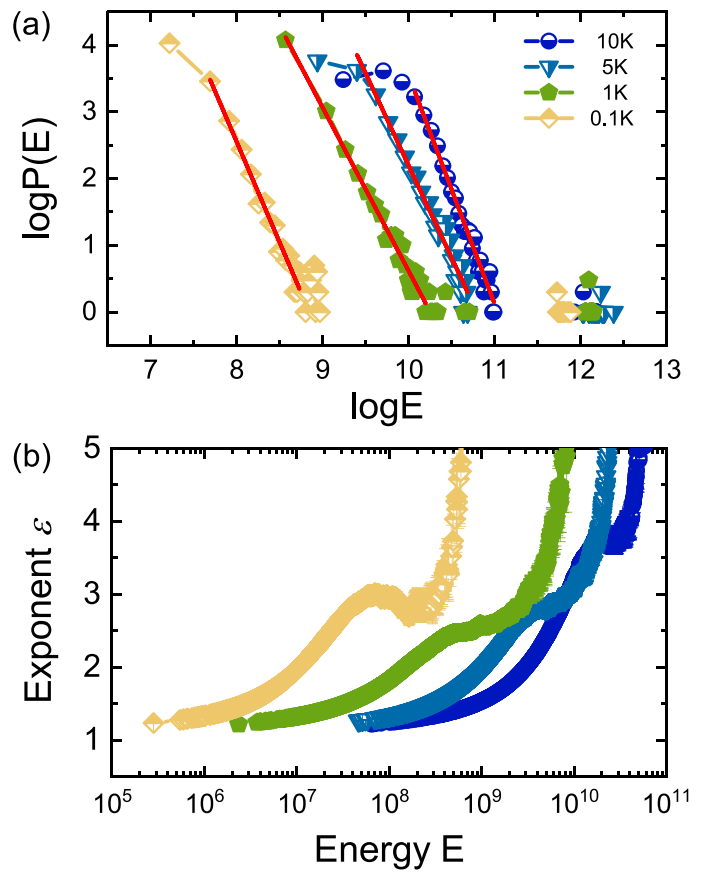

FIG. 2. The probability distribution of the jerk energy $P(E)$ for wave propagation and interaction at temperatures ranging from $0.1 \mathrm{~K}$ to $10 \mathrm{~K}$. It follows the power law at $\mathrm{T}=0.1 \mathrm{~K} \sim 5 \mathrm{~K}$. An exponential cutoff exists at a relatively high temperature $10 \mathrm{~K}$. (b) The maximum likelihood estimations. A plateau is observed for systems with power law distributions.
(ML) estimation with a plateau for power law distributions. The value of plateau is in good accordance with the slope fit in Fig. 2(a).

We further analyze the distribution of event rates of energy jerks $\mathrm{r}_{\mathrm{AS}}$ at $\mathrm{T}=0.1 \mathrm{~K} \sim 5 \mathrm{~K}$, where thermal fluctuations are low. Figure 3(a) shows the variation of $\mathrm{r}_{\mathrm{AS}}$ as a function of time interval with respect to the kink nucleation generated mainshock $\left(t-t_{M S}\right)$ on a logarithmic scale. For very short time periods after the big event, the rate of the superimposed phonons decays with time. It follows a typical Omori law as $\log \left(r_{A S}\right) \sim-p \log \left(t-t_{M S}\right)$, where the exponent $p$ is near 1 . For longer time periods, a plateau appears indicating that the jerky signals are generated at a roughly constant rate. Finally, the rate drops and jerks disappear.

We can characterize two time constants. The first constant $\tau_{\mathrm{I}}$ characterizes the time period over which the Omori law holds. Here, the kink movement and interactions with surfaces emit strong lattice waves. Only one pair of kinks nucleates in small cells. After the kinks bounce back from the surface, additional waves are reflected by the surface and mix with the existing waves. In larger samples, multiple kink nucleation occurs and the phonons from different kinks interacts. Lattice waves have long life times because the damping is small. The phonon interaction still keeps a strong correlation when $\tau<\tau_{\mathrm{I}}$. After the strong correlation period, phonons become scattered and
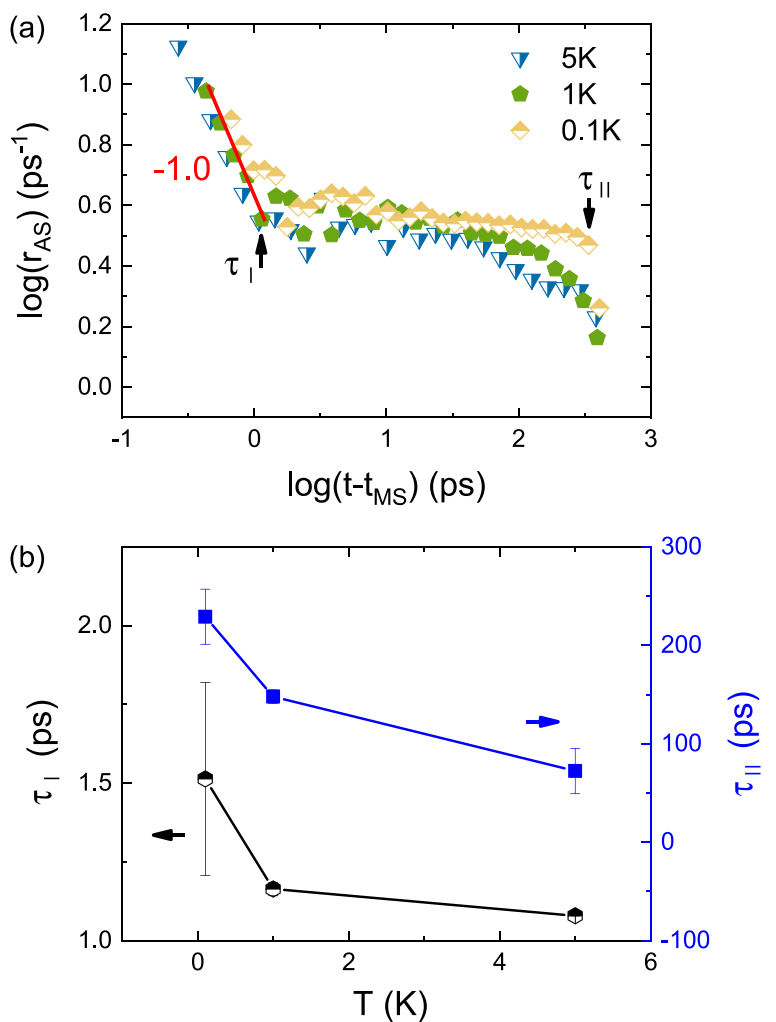

FIG. 3. (a) Event rates of jerk generation as a function of time interval at $T=0.1 \mathrm{~K}$ $\sim 5 \mathrm{~K}$. They follow typical Omori laws with the slope around -1 . Here, two characteristic time constants could be identified as $\tau_{\mid}$and $\tau_{\|}$, which correspond to the starting point and ending point of the plateau. (b) The variation of $\tau_{\mid}$and $\tau_{\|}$as a function of temperature. Both time constants decrease with the increase in temperature. 
uncorrelated. The second constant $\tau_{\text {II }}$ characterizes the time period that the generated phonons annihilated. Figure 3(b) shows the value of $\tau_{\mathrm{I}}$ and $\tau_{\mathrm{II}}$ as a function of temperature. We observe that both the time constants become smaller with increasing temperature. This indicates that the thermal fluctuations add to scattering and phonon damping.

The statistical analysis on the waiting time between jerks at $\mathrm{T}=0.1 \mathrm{~K} \sim 5 \mathrm{~K}$ is shown in Fig. 4. It shows the rescaled distribution of waiting times for different energy thresholds. The distribution of waiting times between jerks is found to follow scaled power laws for a short time, in accordance with the observation of earthquakes and the compression of porous media. The exponents vary in the range of -0.8 $\sim-0.6$. However, at a long time scale, no scaling behavior is observed.

The relaxation of compressed ferroelastics occurs via avalanches. ${ }^{2}$ The avalanche dynamics and the nature of the associated jerks determine the energies and amplitudes of avalanches and have been measured in many systems. ${ }^{3,12,14,22-26}$ The characteristic parameters like energy, amplitude, waiting time duration, and their correlations of jerks were found to be power law distributed. Their exponents are often linked to specific physical models like the mean field model, MF, or the "field integrated mean field model." $27-29$

We have now extended this approach to phonons. Moving kinks emit phonons whenever kinks nucleate, move or annihilate. In each case, phonon clouds are emitted by the kink. These phonons are then scattered by the sample surfaces, domain walls, and other heterogeneities. Their scattering is highly correlated and the question arises whether the initial phonon coherency is maintained and how these phonons interact with thermal phonons. Our results give an answer:

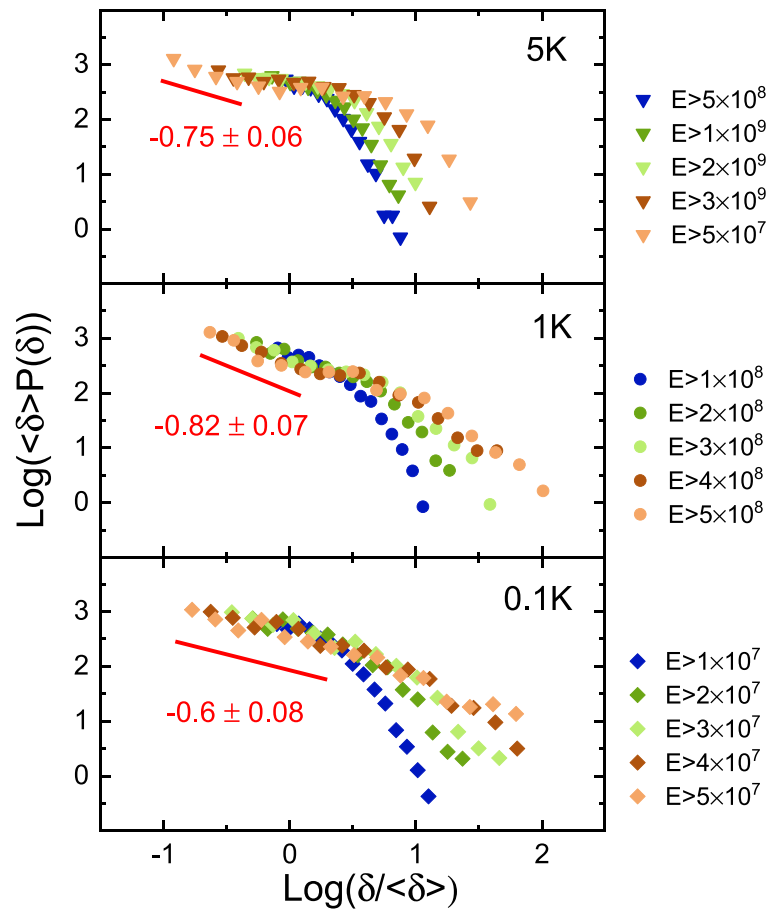

FIG. 4. Rescaled probability distribution of waiting time at $\mathrm{T}=0.1 \mathrm{~K} \sim 5 \mathrm{~K}$. It follows the power law with the exponents $-0.8 \sim-0.6$. The data collapses onto the same line for a short waiting time with different energy thresholds. No scaling behavior exists for a long time period. phonons display similar jerk patterns as the kinks themselves over a large temperature interval near the Vogel-Fulcher point. ${ }^{14,30}$ The power law exponents for phonon energies are rather high with $2.5-3$ at $\mathrm{T}=0.1 \mathrm{~K}-5 \mathrm{~K}$ even when kinks are periodic and do not follow avalanche dynamics. The coherency time is short, namely ca. 1 ps. The equivalent frequency effects are hence expected in the $\mathrm{THz}$ regime and may influence high frequency ferroelectric memory devices as a major loss mechanism. At higher temperature, scattering with thermal phonons becomes predominant and no indication of phononic avalanches was found. The energy distribution follows an exponential distribution as $\mathrm{P}(\mathrm{E}) \sim \exp \left(-\mathrm{E} / \mathrm{E}_{0}\right)$, where $\mathrm{E}_{0}$ is the activation energy (see the supplementary material, Fig. S4). At very low temperatures, quantum effects are expected (for a detailed study, see Ref. 31), which we did not simulate.

Molecular dynamics simulations were carried out to study the avalanche behavior of the kink motion emitted wave (phonons) in ferroelastic materials. The kink nucleates at the twin boundaries upon shear load. Phonon clouds are generated during the kink movement and kink annihilation at the free surface. The propagation and interaction of phonons shows avalanche behavior. At an intermediate temperature below the Vogel-Fulcher temperature, the probability to observe energy jerks follows a power-law spectrum. The jerk generation rate first follows an Omori law, then becomes a constant, and finally drops down. The distribution of waiting time is found to follow a scaled power law. This indicates that the wave interactions first show correlation, and then become uncorrelated by scattering until they finally disappear. As the temperature is higher, the thermal fluctuation has a large damping effect on phonons, where the exponential cut-off becomes dominant in the energy probability distribution.

See the supplementary material for calculations of the local shear strain, activation barrier for kink nucleation, and the profiles of the jerk spectrum at different temperatures.

X.D. and J.S. are grateful to the NSFC (Grant Nos. 51320105014 and 51621063) and the 111 Project (Grant No. BP 2018008) for the financial support. E.K.H.S. is grateful to the EPSRC (No. EP/P024904/1) for the support. S.L. appreciates the support from the NKRDPC (Grant No. 2019YFA0307900).

\section{REFERENCES}

${ }^{1}$ J. P. Sethna, K. A. Dahmen, and C. R. Myers, Nature 410, 242 (2001).

${ }^{2}$ X. Ding, O. Aktas, X. Wang, S. Li, Z. Zhao, L. Zhang, X. He, T. Lookman, A Saxena, and J. Sun, J. Phys.: Condens. Matter 29, 394002 (2017).

${ }^{3}$ F. J. Romero, J. Martín-Olalla, M. C. Gallardo, D. Soto-Parra, E. K. H. Salje, E. Vives, and A. Planes, Phys. Rev. B 99, 224101 (2019).

${ }^{4}$ S. Li, E. K. H. Salje, S. Jun, and X. Ding, Acta Mater. 125, 296 (2017).

${ }^{5}$ X. He, S. Li, X. Ding, J. Sun, S. M. Selbach, and E. K. H. Salje, Acta Mater. 178, 26 (2019).

${ }^{6}$ E. K. H. Salje, D. Xue, X. Ding, K. A. Dahmen, and J. F. Scott, Phys. Rev. Mater. 3, 014415 (2019).

7J. Baró, Á. Corral, X. Illa, A. Planes, E. K. H. Salje, W. Schranz, D. E. SotoParra, and E. Vives, Phys. Rev. Lett. 110, 088702 (2013).

${ }^{8}$ E. K. H. Salje and K. A. Dahmen, Annu. Rev. Conden. Matter Phys. 5, 233 (2014).

${ }^{9}$ T. Utsu, Y. Ogata, and R. S. Matsu'ura, J. Phys. Earth. 43(1), 1-33 (1995).

${ }^{10}$ Á. Corral, Phys. Rev. Lett. 92, 108501 (2004).

${ }^{11}$ E. K. H. Salje, Annu. Rev. Mater. Res. 42, 265 (2012).

${ }^{12}$ D. Soto-Parra, X. Zhang, S. Cao, E. Vives, E. K. H. Salje, and A. Planes, Phys. Rev. E 91, 060401 (2015). 
${ }^{13}$ E. Faran, E. K. H. Salje, and D. Shilo, Appl. Phys. Lett. 107, 071902 (2015).

${ }^{14}$ E. K. H. Salje, X. Ding, Z. Zhao, T. Lookman, and A. Saxena, Phys. Rev. B 83, 104109 (2011)

${ }^{15}$ E. K. H. Salje, S. Li, M. Stengel, P. Gumbsch, and X. Ding, Phys. Rev. B 94 , 024114 (2016).

${ }^{16}$ S. Li, U. Bismayer, X. Ding, and E. K. H. Salje, Appl. Phys. Lett. 108, 022901 (2016).

${ }^{17}$ G. Lu, S. Li, X. Ding, J. Sun, and E. K. H. Salje, Phys. Rev. Mater. 3, 114405 (2019).

${ }^{18}$ G. Lu, S. Li, X. Ding, J. Sun, and E. K. H. Salje, Sci. Rep. 9, 15834 (2019).

${ }^{19}$ X. Wang, E. K. H. Salje, J. Sun, and X. Ding, Appl. Phys. Lett. 112, 012901 (2018).

${ }^{20}$ S. Nose, J. Chem. Phys. 81, 511 (1984).

${ }^{21}$ S. Plimpton, J. Comput. Phys. 117(1), 1 (1995).

${ }^{22}$ R. Niemann, J. Baró, O. Heczko, L. Schultz, S. Fähler, E. Vives, L. Mañosa, and A. Planes, Phys. Rev. B 86, 214101 (2012).
${ }^{23}$ J. Baró, J. Martín-Olalla, F. J. Romero, M. C. Gallardo, E. K. H. Salje, E. Vives, and A. Planes, J. Phys.: Condens. Matter 26, 125401 (2014).

${ }^{24}$ L. Zhang, E. K. H. Salje, X. Ding, and J. Sun, Appl. Phys. Lett. 104, 162906 (2014).

${ }^{25}$ E. Vives, J. Baró, M. C. Gallardo, J. Martín-Olalla, F. J. Romero, S. L. Driver, M. A. Carpenter, E. K. H. Salje, M. Stipcich, R. Romero, and A. Planes, Phys. Rev. B 94, 024102 (2016).

${ }^{26}$ M. Porta, T. Castán, P. Lloveras, A. Saxena, and A. Planes, Phys. Rev. E 98 , 032143 (2018)

${ }^{27}$ K. Dahmen and J. P. Sethna, Phys. Rev. B 53, 14872 (1996).

${ }^{28}$ K. A. Dahmen, Y. Ben-Zion, and J. T. Uhl, Phys. Rev. Lett. 102, 175501 (2009).

${ }^{29} \mathrm{~N}$. Friedman, A. T. Jennings, G. Tsekenis, J. Y. Kim, M. Tao, J. T. Uhl, J. R. Greer, and K. A. Dahmen, Phys. Rev. Lett. 109, 095507 (2012).

${ }^{30}$ X. Ding, T. Lookman, Z. Zhao, A. Saxena, J. Sun, and E. K. H. Salje, Phys. Rev. B 87, 094109 (2013).

${ }^{31}$ S. Kustov, I. Liubimova, and E. K. H. Salje, Phys. Rev. Lett. 124, 016801 (2020). 